\title{
ANÁLISIS E INTERPRETACIÓN DE LOS PERFILES LONGITUDINALES EN LA RED FLUVIAL DEL SUR Y SURESTE DE MALLORCA
}

\author{
A. CAPÓ, C. GARCÍA* \\ Departament de Ciències de la Terra, Universitat de les Illes Balears, \\ 07122 Palma de Mallorca, Balears.
}

\begin{abstract}
RESUMEN: El análisis de los perfiles longitudinales de los ríos a través de la topografía del cauce, derivada de modelos digitales de elevación, es de especial utilidad para la extracción de indicadores geomorfológicos. La interacción entre fuerzas tectónicas y climáticas crea el modelado del paisaje. Cada contexto, definido por sus características climáticas, historia geológica y estado de las fuerzas tectónicas actuales definen un carácter, transitorio o estacionario, a este paisaje, que puede describirse a través de la forma del perfil. En este trabajo se han interpretado 74 perfiles longitudinales de 60 cuencas de drenaje localizadas en el sur y sureste de la isla de Mallorca, con características climáticas semiáridas, tasas de baja actividad tectónica y escasas dataciones cuaternarias. El objetivo es extraer y describir indicadores geomorfológicos y los factores que intervienen. A través de indicadores de forma como la concavidad y convexidad, los resultados muestran que existen zonas en claro estado de inmadurez y desequilibrio con tramos fluviales convexos y otras en cambio con patrones de concavidad en equilibrio dinámico. Se ha podido comprobar que existen 6 zonas con características propias en ocasiones afectadas por una topografía local determinada que obedece a basculamientos y estructuras tectónicas neógenas que perturban ese equilibrio. Las diferentes litologías carbonatadas, con su contraste de comportamiento ante la erosión y los fenómenos de disolución, generan puntos de ruptura en la concavidad y la pendiente, llamados knickpoints. En 31 de los 37 tramos donde el río atraviesa el contacto entre los materiales pliocenos o cuaternarios y las calcarenitas del Tortoniense - Mesiniense se han encontrado knickpoints litológicos. Por otra parte, para el contexto fisiográfico de la zona de estudio, se ha encontrado un umbral en el área de drenaje mínima, entre $2.3 \times 10^{5}$ y $7.2 \times 10^{5} \mathrm{~m}^{2}$, a partir del cual se genera un knickpoint debido a la escorrentía concentrada. El knickpoint en el perfil longitudinal de un río es un factor que genera un estado transitorio en la evolución de la red de drenaje. Además, la reconstrucción de paleoperfiles a partir de knickpoints es útil para determinar bajadas del nivel eustático o saltos de falla bajo determinadas condiciones. En un tramo del sur de la zona de estudio se ha registrado un salto de $30 \mathrm{~m}$ que puede indicar un movimiento vertical de bloques no cartografiado.
\end{abstract}


Analysis and interpretation of the channel topography on the south and southeastern fluvial network of Mallorca

ABSTRACT: The analysis of river longitudinal profiles through the channel topography, derived from digital elevation model, is particularly useful for the extraction of geomorphological indicators. Surface topography reflects the competition and interaction between climatic and tectonic forces and. the shape of the longitudinal river profile contains this information. The modern landscape, defined by its climatic, geological history and current tectonic forces, has a state, transient or steady, that can be described through these indicators. In this work we have analysed 74 longitudinal profiles of 60 drainage basins located in the south and southeast of Mallorca, an area with semi-arid climatic conditions, low rates on tectonic activity and limited Quaternary dating. Through geomorphological indicators such as concavity and convexity, the results show that there are areas in clear state of immaturity and imbalance with a convex river profile and others with a concave profile on a dynamic equilibrium. There are 6 zones with particular characteristics affected by the local topography caused by crustal tilting and Neogene tectonic structures that disrupt that balance. The different carbonate lithologies, with its contrasting response to erosion and dissolution processes, display breakpoints on the concave-upward stream profiles, called knickpoints. In 31 of the 37 streams, lithological knickpoints have been found where the river flows through the contact between materials of the Pliocene and the Quaternary, and the Tortonian and the Messinian calcarenites. Moreover, for the physiographic context of the study area, a threshold has been found in the minimum drainage area, between $2.3 \times 10^{5}$ and $7.2 \times 10^{5} \mathrm{~m}^{2}$, from which a knickpoint is generated due to flow concentration. A knickpoint in the longitudinal profiles is a factor that generates a transient state in the evolution of the drainage network. In addition, reconstruction of paleoprofiles from knickpoints is useful for determining eustatic level changes or fault displacement under certain conditions. A $30 \mathrm{~m}$ displacement has been identified on a stream of the southern area which may indicate an uncharted dip slip fault block displacement.

Key words: perfiles longitudinales, knickpoints, índice de pendiente, área mínima de knickpoint, Mallorca.

Palabras clave: river profiles, knickpoints, channel steepness index, threshold drainage areas, Mallorca.

Enviado el 5 de diciembre de 2013 Aceptado el 12 de febrero de 2014

*Correspondencia: Departament de Ciències de la Terra, Universitat de les Illes Balears, 07122 Palma de Mallorca, Balears. E-mail: celso.garcia@uib.es 


\section{Introducción}

El paisaje terrestre es el producto de la integración temporal de dos factores: la tectónica, que crea la topografía y mantiene el relieve a través del levantamiento de la superficie y las rocas (England y Molnar, 1990; Summerfield, 1991; Whipple, 2004) y el clima, que arbitra en los procesos erosivos que desgastan las áreas montañosas a lo largo del tiempo (Wobus et al., 2003; Allen, 2008). La topografía actual de la superficie terrestre es el producto de la competición e interacción entre las fuerzas tectónicas y climáticas. Mientras que los factores tectónica y clima son difíciles de cuantificar, la topografía terrestre puede ser fácilmente obtenida a través de satélites y fotografía aérea (Wobus et al., 2006). Sin embargo, obtener una comprensión cualitativa de las interacciones entre clima, topografía y tectónica requiere revelar las contribuciones relativas de una serie de complicados procesos superficiales. Este es uno de los temas centrales en la Geomorfología moderna que implica la vinculación entre las tasas en los procesos superficiales y las formas observadas (Dietrich et al., 2003). De hecho, las tasas de erosión generalmente aumentan con el incremento en la pendiente de la ladera, medida como el relieve medio (Ahnert, 1970), hasta una pendiente límite o umbral (Montgomery y Brandon, 2002; Roering et al., 1999) a partir del cual aumenta la frecuencia de deslizamientos y de las tasas de erosión sin un mayor incremento en la pendiente; o también aumentan con la capacidad de los ríos de transportar sedimento o erosionar la roca, a menudo calculado a partir del stream power (Finlayson et al., 2002; Finnegan et al., 2005; Whipple y Tucker, 1999). La tectónica a través de la topografía puede ser particularmente una herramienta poderosa en zonas donde los datos geológicos o geodésicos son escasos (Wobus et al., 2006). En particular, el estudio de los perfiles longitudinales de los ríos ofrece un camino prometedor para explorar la relación entre la incisión fluvial y la tectónica (Howard et al., 1994; Hovius, 2000). Los perfiles longitudinales han sido ampliamente utilizados como indicadores de la evolución geomorfológica (e.g. Duvall et al., 2004; Bishop, 2007; Goldrick y Bishop, 2007). Estos tienden hacia una forma cóncava que facilita el transporte del sedimento y la erosión del lecho fluvial (Hack, 1957) y han sido considerados durante mucho tiempo como un estado normal, de equilibrio dinámico, en la evolución del perfil del río (Snow y Slingerland, 1987; Whipple y Tucker, 1999; Whipple, 2001). La evolución en el cauce y su incisión, que está controlada por las condiciones climáticas, litológicas y tectónicas, y por las fluctuaciones en el nivel de base, no siempre desarrolla una concavidad uniforme asociada con un estado de equilibrio dinámico. De hecho, algunos ríos no pueden alcanzar la concavidad y a lo largo del perfil longitudinal se encuentran tramos en desequilibrio con mucha pendiente, denominados convexidades, rupturas o knickpoints que reflejan una localizada incisión en el lecho (Gardner, 1983). Es decir, el sistema fluvial responde a una perturbación, un forzamiento tectónico, a través de la generación aguas arriba de knickpoints, o cambios en la pendiente del perfil del río, cuya forma y comportamiento depende de la naturaleza de la perturbación y de la mecánica de la incisión fluvial. El knickpoint puede considerarse como el límite migratorio entre la región aguas abajo que se adapta al nuevo forzamiento y la región aguas arriba que retiene las características del estado pre-existente o anterior (Whipple et al., 2011). 
En este trabajo presentamos el análisis e interpretación de perfiles longitudinales en cuencas de drenaje localizadas en el sur y sureste de la isla de Mallorca. Se ha utilizado un modelo digital de elevación (MDE) de $2 \mathrm{~m}$ de resolución horizontal para evaluar en detalle la topografía del perfil longitudinal con el objetivo de determinar la geometría y distribución de los perfiles por zonas, calcular el índice de pendiente e identificar los knickpoints a lo largo del perfil longitudinal. Finalmente, se establece el área mínima o superficie de cuenca de drenaje a partir de la cual se desarrollan los knickpoints para el contexto litológico y climatológico de la zona de estudio.

\section{2. Área de estudio}

La isla de Mallorca es un segmento del cinturón de pliegues y cabalgamientos producto de la evolución tectónica del mediterráneo occidental. La estructura geológica actual de la isla es el resultado de una evolución compleja que ha dado como resultado un conjunto de horst y grabens, que se disponen de manera alternativa dando lugar a las sierras y llanos que conforman la actual orografía de la isla. Sobre los materiales plegados del Mesozoico descansan los depósitos del Mioceno superior, Plioceno y Pleistoceno que forman plataformas casi horizontales alrededor de las sierras.

La red hidrográfica en la isla de Mallorca está muy influenciada por el relieve, la litología calcárea y el clima mediterráneo. Los cursos fluviales tienen una escorrentía superficial esporádica muy marcada por las precipitaciones y dependiente de las aportaciones de las aguas subterráneas en un terreno calcáreo, mayoritariamente permeable. El resultado es un régimen hidrológico efímero o estacional en aquellos cursos vinculados con las aportaciones de fuentes y manantiales. En la zona de estudio (Fig. 1), la red fluvial se desarrolla sobre unas plataformas casi horizontales, denominadas marinas de Llucmajor, Santanyí y Llevant, rodeadas por un relieve bajo en las cabeceras, en torno a los $540 \mathrm{~m}$ en el Puig de Randa y entre los 300 y 500 m en las sierras de Llevant. Los cursos son de corto recorrido, encajados como cañones fluviales, con un comportamiento torrencial condicionado por la karstificación y la estructura. Entre la marina de Llucmajor y la de Santanyí se desarrolla la depresión de Campos, con cursos fluviales de mayor recorrido, muchas veces no definidos, y que convergen en un humedal separado del mar por un cordón dunar. En esta zona meridional y sudoriental, de carácter semi-árido, las precipitaciones medias anuales oscilan entre los 300 y $500 \mathrm{~mm}$, con precipitaciones máximas diarias entre 110 y $150 \mathrm{~mm}$ para un periodo de retorno de 50 años. Las crecidas son súbitas y de corta duración, con tiempos de concentración breves, condicionados por el episodio de precipitación y con caudales punta que pueden superar los $500 \mathrm{~m}^{3} \mathrm{~s}^{-1}$ como los registrados el septiembre de 1989 (Grimalt y Rodríguez-Perea, 1989).

La amplia zona de estudio ha sido elegida por tener características homogéneas tales como la topografía, con pocos relieves importantes; zonas relativamente llanas de superficie estructural y una historia geológica prácticamente común en toda el área. No obstante, la zona presenta heterogeneidades suficientes para que su análisis no pueda realizarse por completo comparando características comunes a todos los tramos fluviales, sino que deben ser agrupados para, en primera instancia, obtener el comportamiento de cada grupo y, posteriormente, comparar con el resto. 


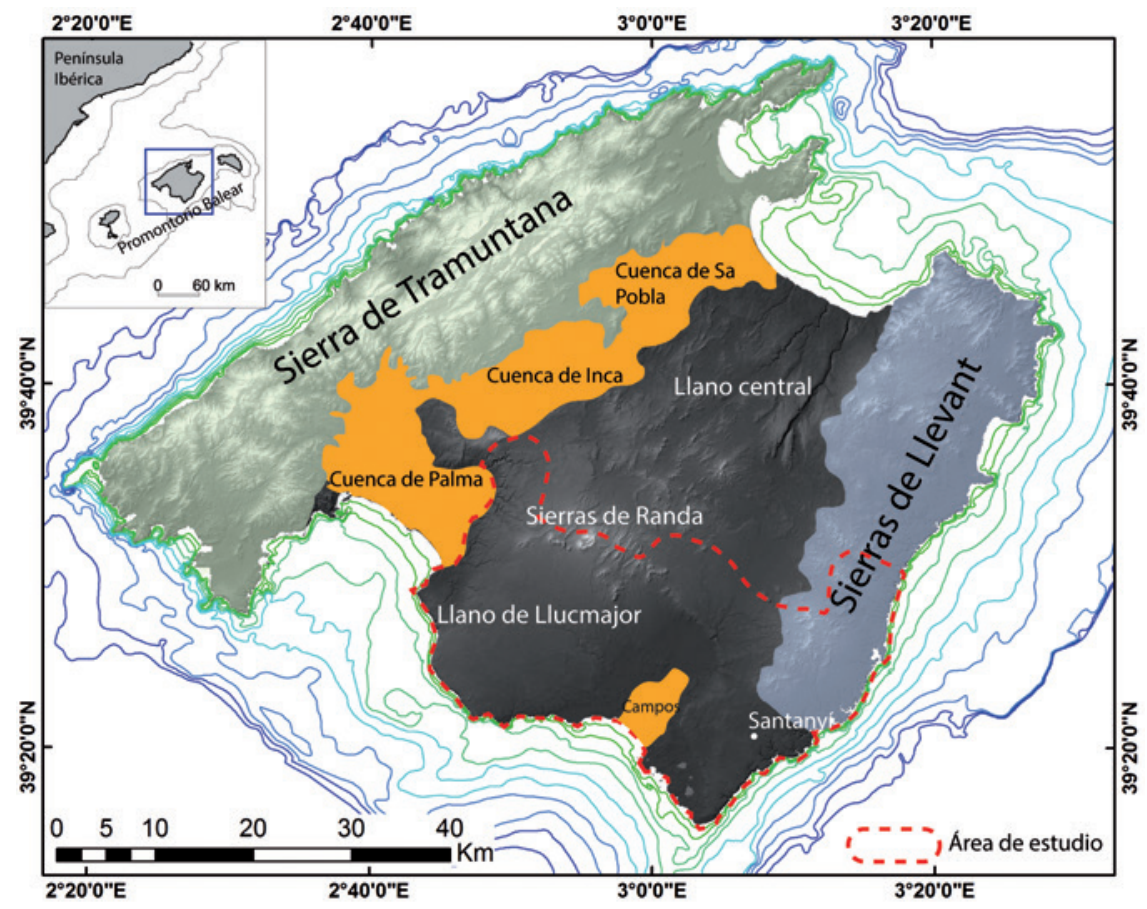

Figura 1. Mapa de localización de la isla de Mallorca y la zona de estudio.

Se han estudiado un total de 60 cuencas de drenaje con sus respectivos afluentes para un total de 74 tramos fluviales, a lo largo del sur y sureste de la isla de Mallorca. Las cuencas tienen superficies que van desde los $2.8 \times 10^{5} \mathrm{~m}^{2}$ hasta $\operatorname{los} 3.8 \times 10^{8} \mathrm{~m}^{2}$. El área de estudio se ha dividido en 6 zonas (Fig. 2) en base a criterios geomorfológicos como la orientación del drenaje, la topografía, los patrones de drenaje fluvial así como sus superficies, el tipo de costa donde desembocan al mar, la litología del substrato y su contexto geológico local. Esto facilita la descripción y comprensión de las diferentes configuraciones fluviales, los fenómenos de origen geológico y las características geomorfológicas presentes en el área.

\section{Métodos}

\subsection{Construcción de un modelo digital de elevación (MDE)}

A partir del MDE de 5 metros del Instituto Geográfico Nacional y la topografía 1:5000 se ha construido un modelo digital de elevación corregido y adaptado especialmente para los análisis hidrológicos. La resolución espacial XY del modelo resultante es de $2 \mathrm{~m}$ y su resolución en la vertical $\mathrm{Z}$ es de $5 \mathrm{~m}$. El modelo fue interpolado mediante la herramienta topo to raster incluida en el software ArcGis 10.1, la cual es un método de interpolación diseñado específicamente para crear un MDE hidrológicamente correcto y está basada en el programa ANUDEM desarrollado por Hutchinson (1989). 


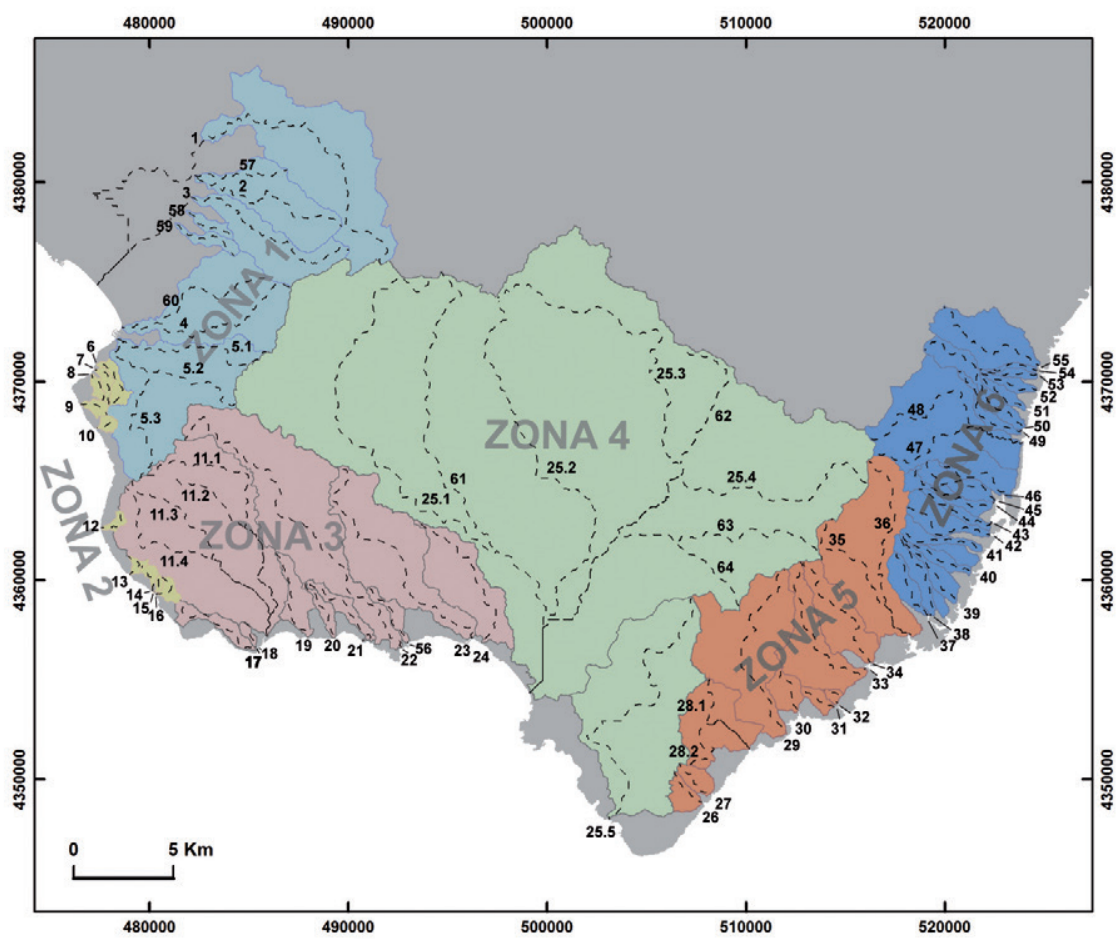

Figura 2. Mapa del sur y sureste de Mallorca con la delimitación por zonas, la red fluvial (discontinuo) y su numeración por tramos.

\subsection{La red de drenaje}

El análisis hidrológico de la red de drenaje se ha realizado con el módulo Hidrología de ArcGis 10.1. En este caso, las direcciones del flujo sobre el MDE fueron obtenidas con el algoritmo de flujo D8 basado en la identificación del píxel más bajo dentro de los ocho píxeles que rodean al píxel considerado. Así, se puede comparar la organización de la red de drenaje en relación a los dominios topográficos y geológicos, entender la distribución espacial de los ríos, su relación con las estructuras tectónicas y litologías, y analizar la geometría de los perfiles longitudinales.

\subsection{Los perfiles longitudinales y la pendiente}

Para cada cuenca se extrajo el valor de elevación en función de la distancia a la cabecera de los ríos principales y sus afluentes más importantes. Se utilizó el programa Origin v8 para representar las elevaciones de los ríos en función de la distancia a la cabecera. El objetivo es analizar las características y particularidades de los mismos, sobre- 
todo su pendiente y uniformidad, para posteriormente compararlos entre ellos y obtener información sobre la red de drenaje y su relación con el contexto donde se emplazan. Una vez generado cada perfil longitudinal se ajusta una curva ideal que describe el perfil longitudinal de un río en dos dimensiones para tramos de pendiente similar (Whipple y Tucker, 1999; Whipple, 2001):

$$
z(x)=k_{s} k_{a}^{-\theta}(1-h \Theta)-1\left(L^{1-h \theta}-x_{c}{ }^{1-h \theta}\right)+z(L)
$$

donde $\mathrm{z}$ es la elevación, $\mathrm{x}_{\mathrm{c}}$ es la distancia desde la divisoria a la cabecera; $\mathrm{L}$ es la distancia hasta la desembocadura, $\mathrm{k}_{\mathrm{s}}$ es el índice de pendiente, $\theta$ el índice de concavidad, $\mathrm{k}_{\mathrm{a}}$ el coeficiente de Hack y h el exponente de Hack; bajo las condiciones de $\mathrm{h} \theta \neq 1 ; \mathrm{x}_{\mathrm{c}} \leq$ $\mathrm{x} \leq \mathrm{L}$. El propósito de aplicar esta fórmula es extraer el valor del índice de pendiente como única variable libre en los diferentes ríos para poder realizar una comparación entre ellos, fijando $\theta=0.45 ; \mathrm{k}_{\mathrm{a}}=6.7 ; \mathrm{h}=1.67$ (Whipple y Tucker, 1999). De esta manera el índice de pendiente pasa a ser normalizado $\left(\mathrm{K}_{\mathrm{sn}}\right)$.

Según la teoría del stream power (Howard et al., 1994), el índice de pendiente de los ríos $\left(\mathrm{k}_{\mathrm{s}}\right)$ en equilibrio estático, donde la erosión compensa el levantamiento de las rocas, es función de la tasa de levantamiento relativo y de la resistencia de la roca a la erosión que depende tanto de la litología como de las condiciones climáticas. Esta teoría asume que los ríos inciden sobre un sustrato homogéneo y bajo condiciones climáticas y de levantamiento homogéneas en el espacio y constantes en el tiempo (Whipple, 2004). El análisis de casos naturales muestra que por motivos de cambios de nivel de base, evolución del orógeno, fenómenos de captura, heterogeneidades en los valores de erosión a través del perfil de un río, éste puede no presentar un ajuste perfecto a una sola curva ideal y es necesario ajustar por tramos varias curvas, lo que da diferentes valores de $\mathrm{K}_{\mathrm{sn}}$ para cada tramo.

La representación gráfica de los índices de $\mathrm{K}_{\mathrm{sn}}$ se ha realizado con una escala de color que está normalizada respecto a la media de cada zona en lugar de toda el área de estudio con el fin de maximizar la información representada en cada figura. Así, la escala de colores no será extrapolable entre zonas pero ofrecerá una mayor resolución entre los tramos de cada una de ellas.

\subsection{Los knickpoints}

La morfología del knickpoint está marcada por un cambio distintivo en la pendiente del cauce y puede ser identificado a través de las representaciones gráficas de los perfiles longitudinales de elevación y distancia, entre la pendiente y el área, o la pendiente y la distancia (Goldrich y Bishop, 2007) localizándose puntos donde se produce una ruptura en la uniformidad del perfil. Esta uniformidad siempre trunca la continuidad en la concavidad del perfil y de su índice de pendiente normalizado $\left(\mathrm{k}_{\mathrm{sn}}\right)$. Así pues, su localización consta de una fase visual y una fase analítica. Su existencia se debe a la respuesta del río ante perturbaciones tales como estructuras geológicas que provoquen una heterogeneidad en el levantamiento local de la superficie, diferentes litologías que provoquen un contraste en las tasas de erosión, la existencia de fenómenos de captura 
fluvial que provocan un aumento repentino del área de drenaje y por último los movimientos relativos del nivel del mar, especialmente los de mayor amplitud.

\section{Resultados y discusión}

\subsection{Zona 1: Cuenca de Palma}

Los tramos fluviales comparten una misma dirección de drenaje noroeste, condicionada por la pendiente de una superficie estructural que corresponde a una plataforma continental emergida de edad miocena ligeramente basculada y tapizada por sedimentos pliocenos y cuaternarios (Fig. 3). La longitud de los tramos está comprendida entre 9 y $17 \mathrm{~km}$ y salvan desniveles de hasta $300 \mathrm{~m}$. Un hecho remarcable y recurrente en todas las zonas, excepto la 3 y 4, es que se aprecia una incisión de magnitud métrica, en ocasiones decamétrica, con valles muy verticales, una sinuosidad elevada y la divisoria de aguas situada en una topografía prácticamente llana. Esta configuración es típica de los cañones fluviales y puede apreciarse en mayor medida en los ríos situados al norte de esta zona.

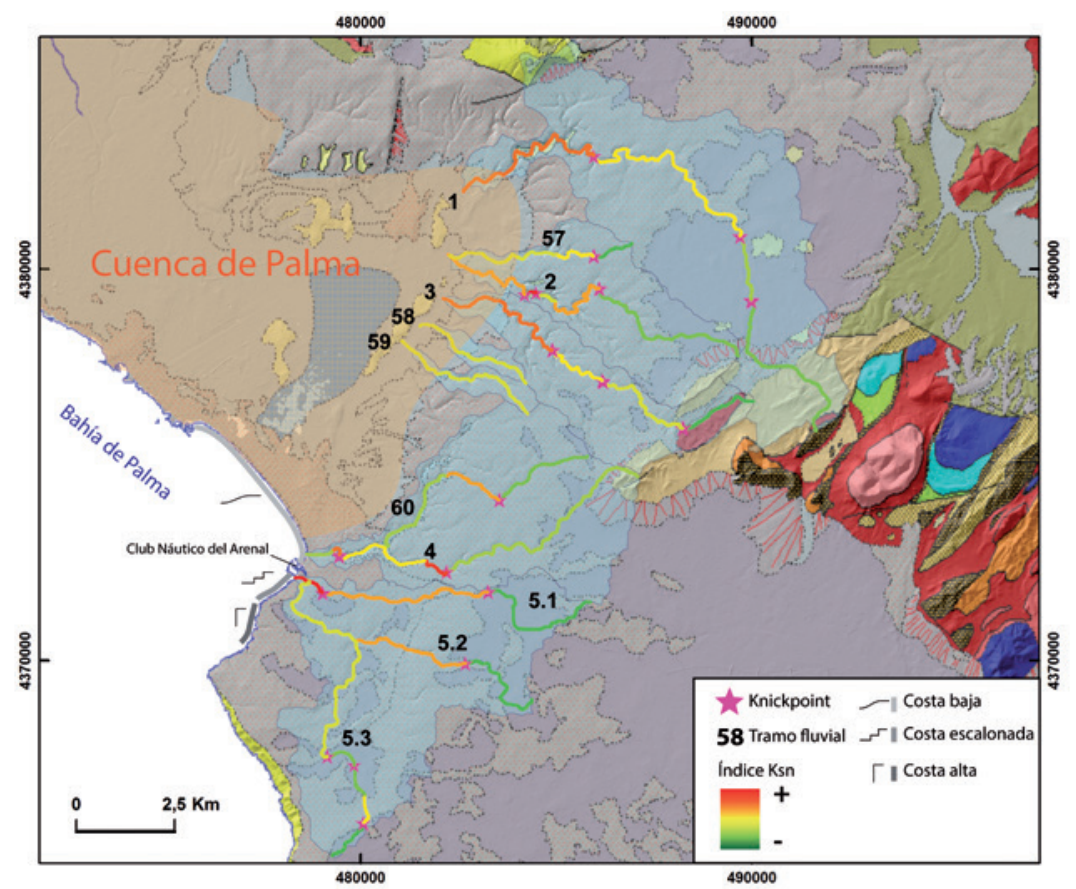

Figura 3. Mapa geológico detalle de la zona 1 (fuente: IGME) correspondiente a los ríos que drenan hacia la cuenca de Palma con los trazados de la red fluvial y su gradación según su índice de $K_{s n}$, la localización de los knickpoints y el tipo de costa.

El lector puede remitirse a la leyenda del mapa del IGME.

Una parte de los tramos han sido seleccionados hasta el nivel de base de la cuenca de Palma en lugar del mar, dado que allí adquieren un gradiente suficientemente bajo 
como para dejar de considerar su acción erosiva. La otra parte de los tramos mantienen un gradiente suficiente como para permitir, de manera normal, su análisis hasta el nivel del mar. En este último caso, el tipo de costa es de perfil bajo o excepcionalmente escalonado de bajo relieve.

Las litologías por la que transcurren estos tramos son principalmente limos y arcillas con cantos del Cuaternario y areniscas poco cementadas de origen litoral del Plioceno. Dos de los tramos ( 1 y 3 ) drenan en su cabecera los relieves más occidentales del macizo de Randa, que corresponden a materiales sin y post orogénicos de edad paleógena. Estos materiales son calizas, limos y arcillas del Oligoceno que cabalgan sobre margas y areniscas del Mioceno inferior (Gelabert, 1998). En algunos casos, siendo más evidente en el tramo 1, la particular incisión en forma de cañón permite que el río erosione por completo el Plio-cuaternario e incida directamente sobre las calizas del Oligoceno, haciéndolas aflorar en el interior del cañón.

El análisis de los knickpoints a partir de los perfiles longitudinales muestra que el tramo 3 presenta un knickpoint de origen litológico en su cabecera en el contacto entre los materiales sinorogénicos y los postorogénicos. Los cañones que llegan a erosionar sobre las calizas del Oligoceno reflejan un knickpoint en dicho punto, hecho que plantea la posibilidad de que haya diferentes tasas de erosión condicionadas por fenómenos cársticos y correspondan también a knickpoints de origen litológico. El resto de knickpoints no parecen obedecer a ningún contraste de competencia entre litologías ni a estructuras tectónicas y pueden indicar que sean la respuesta a cambios eustáticos o procesos de captura fluvial.

\subsection{Zona 2: Acantilados de la marina de Llucmajor}

Estos cursos fluviales (Fig. 4) tienen la particularidad de drenar hacia una desembocadura situada en tramos de costa alta, que comprenden acantilados de entre 8 y $120 \mathrm{~m}$, donde se alternan tramos de perfil vertical puro con tramos de perfil cóncavo de elevado gradiente. Son tramos de recorrido medio de entre 1.1 y $3.2 \mathrm{~km}$ y áreas de drenaje muy reducidas, salvando desniveles de hasta $130 \mathrm{~m}$. Transcurren por litologías que en la cabecera corresponden a los limos y arcillas con cantos del Cuaternario y areniscas poco cementadas de origen litoral del Plioceno. En su parte final y coincidiendo con el aumento de gradiente cercano a la costa que comprende valores de entre 35 y 80 grados, éstos pasan a incidir sobre la capa subyacente de calcarenitas bioclásticas, arrecifales y oolíticas del Tortoniense-Mesiniense, las cuales dominan durante la mayor parte de los acantilados de la zona.

Todos los tramos de esta zona presentan como mínimo dos knickpoints en su recorrido. Los tramos situados más al norte presentan un tercer knickpoint hacia su cabecera con una marcada alineación entre ellos NE-SW. El siguiente nivel de knickpoints, de origen litológico y común en todos los tramos, corresponde al contacto entre los niveles Pliocenos con los Miocenos arrecifales más porosos, a excepción de los tramos 6, 7 y 8 donde se presenta ese límite. El tercer y último nivel de knickpoints coincide en todos los tramos con la ruptura de la pendiente que generan los acantilados marinos. Esta ruptura, de origen estructural (Gelabert et al., 2007), está fosilizada en varios tramos y en su base por dunas pleistocenas, ofreciendo un marco temporal sobre el que situar los knickpoints encontrados, su dinámica y clasificarlos como de origen estructural. 


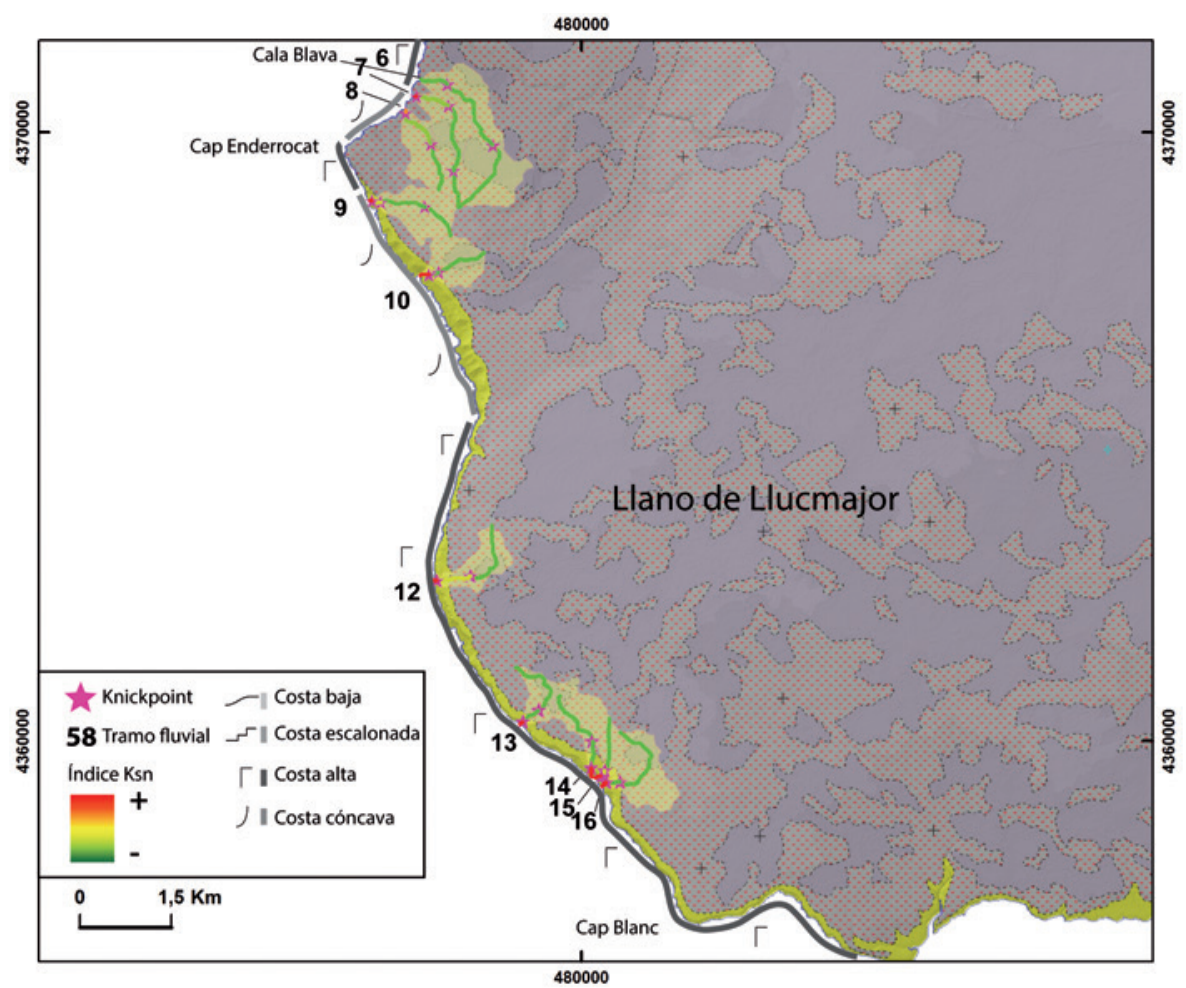

Figura 4. Mapa geológico de la zona 2 (fuente: IGME) correspondiente a los ríos que inciden sobre los acantilados de la costa de Llucmajor, con los trazados de la red fluvial y su gradación según su índice de $K_{s n}$, la localización de los knickpoints y el tipo de costa. El lector puede remitirse a la leyenda del mapa del IGME.

\subsection{Zona 3: Cala Pi y Sa Ràpita}

Todos los tramos de esta zona, al igual que en la Zona 1, comparten una misma dirección de drenaje, aunque en este caso con dirección sureste (Fig. 5). También comparte con la Zona 1 que el drenaje, prácticamente paralelo entre cuencas, obedece a un suave relieve estructural de plataforma Miocena emergida y ligeramente basculada. La mayoría de los tramos tienen una longitud entre 10 y $20 \mathrm{~km}$ y salvando desniveles de entre 50 y $150 \mathrm{~m}$. Al contrario que la zona 1, éstos no presentan una incisión en forma de cañones a excepción de sus tramos finales, como el cañón de Cala Pi que corresponde al tramo 11, coincidiendo con una red más desarrollada y una mayor área de drenaje de la zona. La divisoria de aguas entre la Zona 1 y la Zona 3, de marcada forma rectilínea, define la charnela que separa ambos lados de la plataforma basculada. Se desconoce actualmente si este hecho ha sido provocado por plegamiento o por basculamiento de bloques por medio de fallas. 


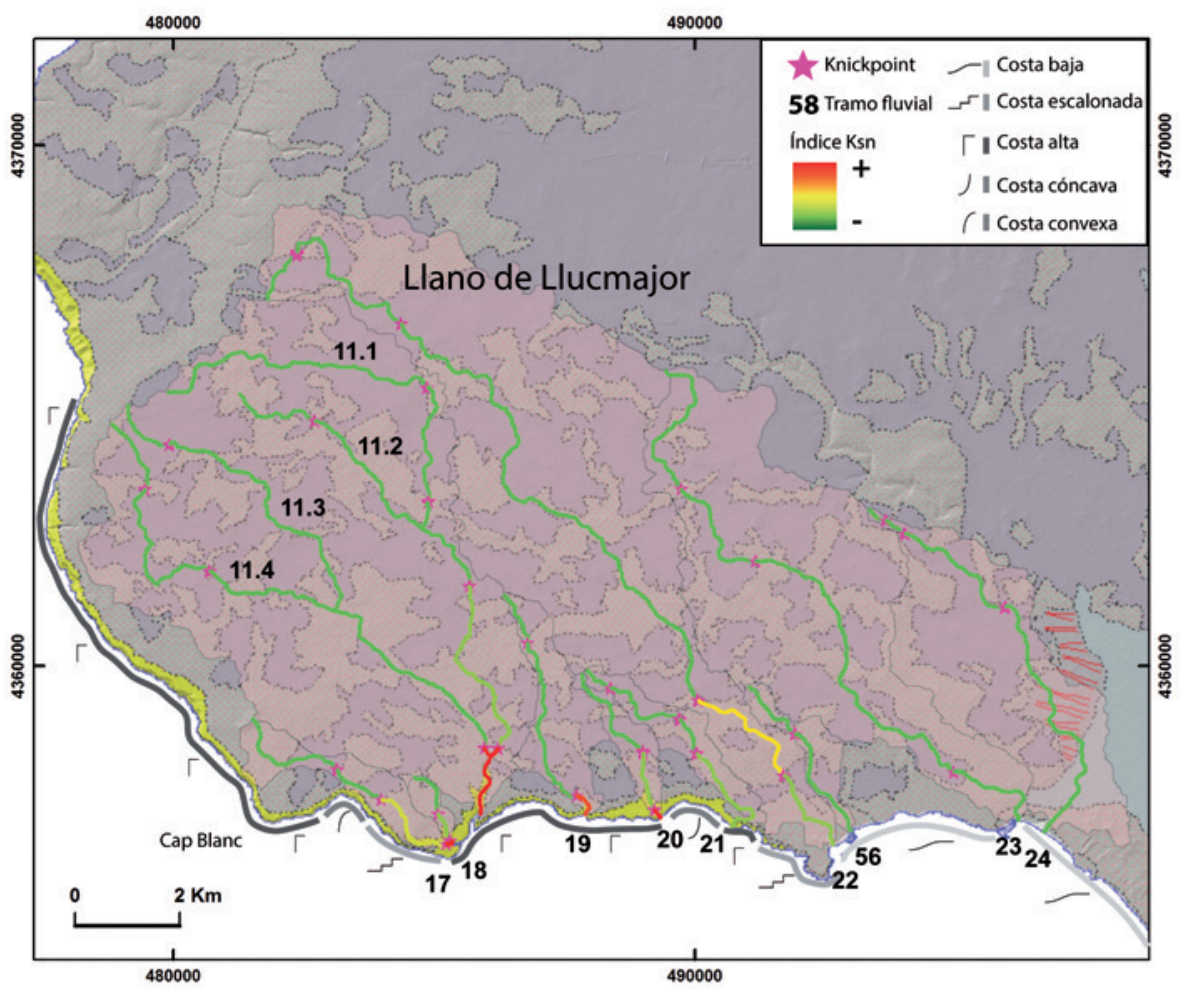

Figura 5. Mapa geológico de la zona 3 (fuente: IGME) correspondiente a los ríos que inciden sobre el llano de Llucmajor, con los trazados de la red fluvial y su gradación según su índice de $K_{s n}$, la localización de los knickpoints y el tipo de costa. El lector puede remitirse a la leyenda del mapa del IGME.

Las litologías por las que transcurren en su mayor parte corresponden a los materiales del Cuaternario y Plioceno. Como ocurre en la Zona 2, en su parte final y cercano a la costa, éstos pasan a incidir sobre las calcarenitas bioclásticas, arrecifales y oolíticas, las cuales siguen dominando los afloramientos de costa alta en la zona.

Los knickpoints detectados se pueden agrupar en dos grupos. En las partes altas y vinculados con las zonas de pendiente estructural con poco relieve, aparecen pequeños knickpoints sin a priori poder clasificar su origen, ya que no presentan un patrón en su distribución ni se corresponden a variaciones en la litología ni atraviesan estructuras tectónicas que estén actualmente cartografiadas. El segundo grupo corresponde a knickpoints cercanos a la costa y que implican un aumento considerable del índice de $\mathrm{K}_{\mathrm{sn}}$ por encima de valores de 60 y hasta 200, muy por encima de la media del área de estudio. Estas rupturas muestran que los tramos han llegado a incidir sobre las capas más porosas de calcarenitas arrecifales y bioclásticas del Tortoniense como ocurre en las zonas anteriores. Se trata de knickpoints de origen litológico. 


\subsection{Zona 4: Cuencas de Llucmajor, Campos y Ses Salines}

Los tramos fluviales presentan cabeceras ubicadas en diferentes contextos (Fig. 6) pero todos fluyen hacia un mismo punto y comparten un mismo nivel de base, a excepción del tramo 25.5. La longitud de los tramos está comprendida entre los 17 y $35 \mathrm{~km}$ y la elevación entre las cabeceras y el mar es de entre 250 y 500 m. Los tramos más occidentales tienen su cabecera en la Sierra de Randa y los tramos más orientales en las Sierras de Levante, cubriendo así una extensa área de drenaje total. Los primeros inciden sobre materiales calcáreos mayormente sinorogénicos Miocenos ligeramente deformados mientras que los segundos inciden sobre margas, dolomías y carbonatos preorogénicos del Mesozoico.

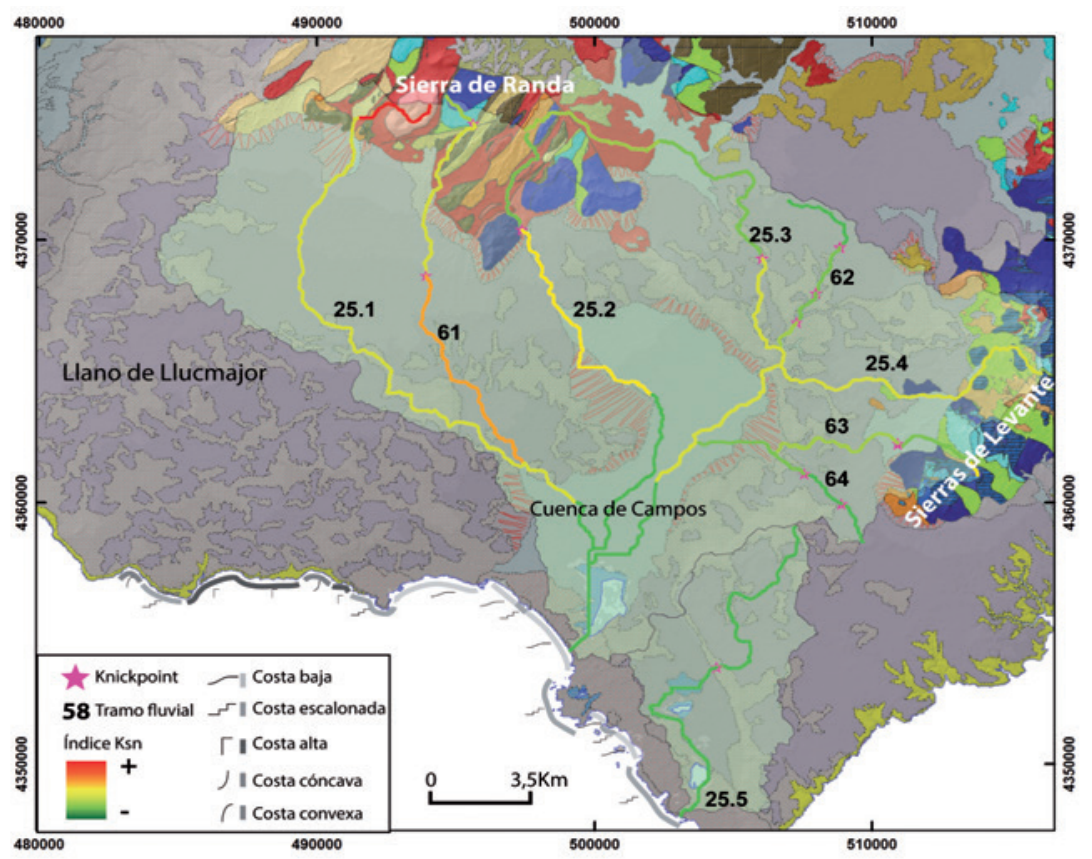

Figura 6. Mapa geológico de la zona 4 (fuente: IGME) correspondiente a los ríos que drenan hacia la cuenca de Campos, con los trazados de la red fluvial y su gradación según su índice de $K_{s n}$, la localización de los Knickpoints y el tipo de costa. El lector puede remitirse a la leyenda del mapa del IGME.

Las litologías, una vez fuera de dichos relieves, vuelven a ser los materiales Pliocenos y Cuaternarios que tapizan la plataforma. En este caso ningún tramo llega a incidir sobre los materiales arrecifales del Tortoniense y en sus tramos finales todos los ríos confluyen en la cuenca de Campos, un humedal prácticamente al nivel del mar que actúa como nivel de base, antes de llegar al mar.

Los índices de pendiente $\left(\mathrm{K}_{\mathrm{sn}}\right)$ muestran que, a diferencia de las zonas anteriores, hay cabeceras con un índice elevado, con valores $>20$, situadas en zonas de relieve mon- 
tañoso. Los índices de pendiente disminuyen por debajo de 20 cerca del contacto entre los materiales pre y sinorogénicos y los Pliocenos y Cuaternarios de las zonas de menor topografía, siguiendo un patrón cóncavo de equilibrio dinámico. A partir de este punto el comportamiento es similar a la zona 3 , fluyendo a través del relieve suave y uniforme, en este caso hacia la cuenca de Campos.

Por otra parte, los knickpoints que se ubican en las zonas de cabecera son debidos a la heterogeneidad de litologías y estructuras de los complejos sinorogénicos. Otros, de menor salto, se encuentran en zonas de topografía suave que no parecen coincidir con cambios litológicos ni ningún otro fenómeno que en primera instancia nos permita clasificarlos. Finalmente, en el tramo 25.5 se encuentra un knickpoint de salto de orden decamétrico que separa dos tramos con el mismo índice de pendiente. Dado que no parece existir ningún contraste litológico que justifique dicho salto es probable que o bien se trate de un knickpoint asociado a un descenso brusco del nivel del mar o bien a un movimiento vertical de bloques causado por una falla. En cualquier caso, se ha calculado el salto entre la parte superior e inferior extrapolando el perfil superior según su pendiente original extraída del perfil longitudinal y comparándolo con la altitud del actual nivel de base (Fig. 7). El salto es de $30 \mathrm{~m}$ y tanto aguas arriba del knickpoint como aguas abajo se encuentran eolianitas de origen litoral del Plioceno. El mapa geológico actual no cartografía ninguna falla, aunque se observa que los materiales Pliocenos que afloran tanto aguas arriba como abajo del resalte están cubiertos parcialmente por material Cuaternario imposibilitando a priori la detección de alguna fractura. Si el knickpoint fuera debido a una bajada brusca del nivel del mar entre el Plioceno y el Cuaternario se encontrarían más situaciones similares en los perfiles longitudinales restantes que desembocan en el mar, hecho que no se produce, no al menos de forma tan clara y evidente.

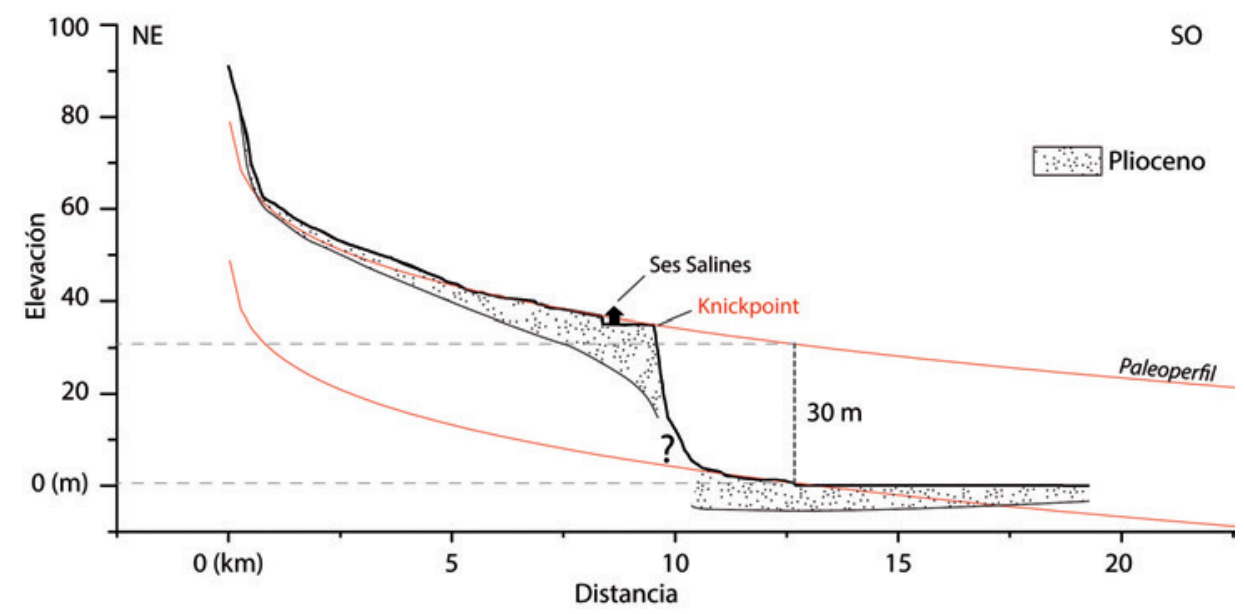

Figura 7. Representación gráfica del perfil longitudinal del tramo 25.5 y la extrapolación del paleoperfil en las dos alturas: superior e inferior al knickpoint así como el salto resultante entre bloques. El entramado corresponde a materiales del Plioceno. 


\subsection{Zona 5: Levante bajo}

Los tramos de esta zona del levante mallorquín (Fig. 8) se caracterizan por tener un drenaje con dirección sureste, de una longitud comprendida entre 3 y $12 \mathrm{~km}$, desembocando sobre una costa relativamente alta. Algunos tramos tienen como cabecera la vertiente sureste de las Sierras de levante, entre 200 y 300 m de altitud y hacia el sur sobre un relieve con poca pendiente correspondiente a la plataforma emergida tapizada por los sedimentos Cuaternarios. En este caso los afloramientos del Plioceno quedan reducidos a los dos tramos más meridionales.

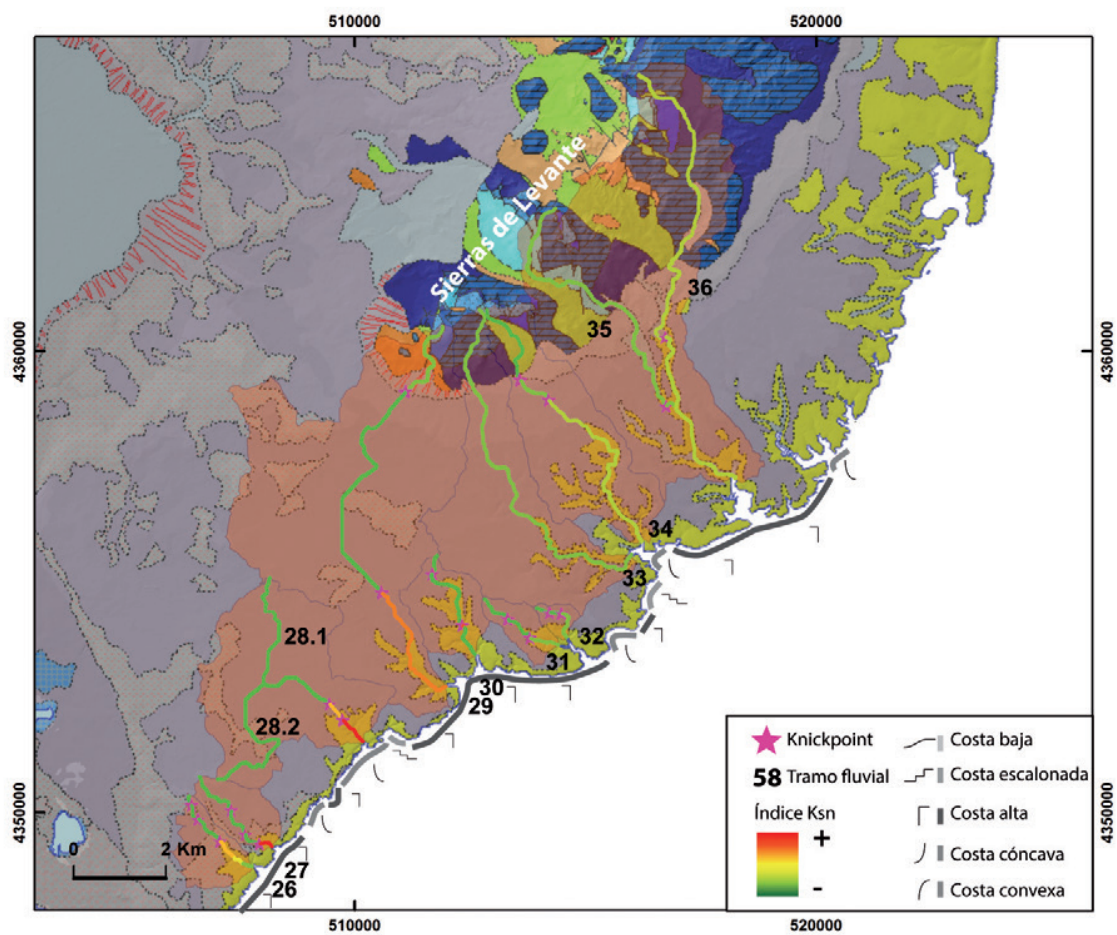

Figura 8. Mapa geológico de la zona 5 (fuente: IGME) correspondiente a los ríos que inciden en la parte más meridional del levante de Mallorca, con los trazados de la red fluvial y su gradación según su índice de $K_{s n}$, la localización de los knickpoints y el tipo de costa. El lector puede remitirse a la leyenda del mapa del IGME.

Otra característica es que aunque todos transcurren con un drenaje más o menos paralelo, algunos tramos tienen su cabecera situada en la topografía elevada y otros simplemente aparecen en medio de una topografía más plana cercana a la costa. De modo generalizado, estos ríos llegan a incidir en los sedimentos arrecifales de plataforma y generan cañones cercanos al mar que pueden llegar a tener una incisión de entre 10 y $20 \mathrm{~m}$. Esto provoca que desemboquen con una pendiente relativamente suave y uniforme rodeados de costa alta. Es el accidente geográfico denominado cala (Rosselló i Verger, 2007) y que tiene su mayor representación en las zonas de levante bajo y alto. 
Las litologías en las cabeceras de las Sierras de Levante son carbonatos, margas y dolomías del Jurásico y Cretácico en su mayor parte. En los tramos intermedios transcurren por superficies tapizadas por sedimento aluvial Cuaternario y en las partes cercanas al mar inciden sobre calcarenitas arrecifales y oolíticas del Tortoniense.

El análisis de los knickpoints muestra que en 10 de los 11 tramos hay un knickpoint de origen litológico asociado al contacto Cuaternario-Tortoniense y en los dos tramos donde aflora Plioceno también se produce entre los contactos Cuaternario-Plioceno y Plioceno-Tortoniense. Los tramos que drenan los relieves de las Sierras de Levante no presentan ningún knickpoint de origen estructural.

\subsection{Zona 6: Levante alto}

Esta zona se ha separado de la anterior debido a su patrón de distribución: los tramos son más paralelos y con una separación entre ellos mucho menor, con 18 tramos en apenas $10 \mathrm{~km}$, lo que resulta en una separación media de unos $600 \mathrm{~m}$ entre divisorias. Tienen longitudes comprendidas entre 3 y $12 \mathrm{~km}$. Las cabeceras tienen su origen en las Sierras de Levante, en elevaciones de entre 200 y $400 \mathrm{~m}$, aguas abajo transcurren por una zona llana de material Cuaternario y desembocan finalmente en forma de calas (Fig. 9). El contexto litológico es idéntico al de la zona 5: carbonatos, margas y dolomías del Jurásico y Cretácico en las cabeceras, sedimento aluvial Cuaternario en los tramos medios y en las partes cercanas al mar transcurren sobre las calcarenitas arrecifales y oolíticas del Tortoniense.

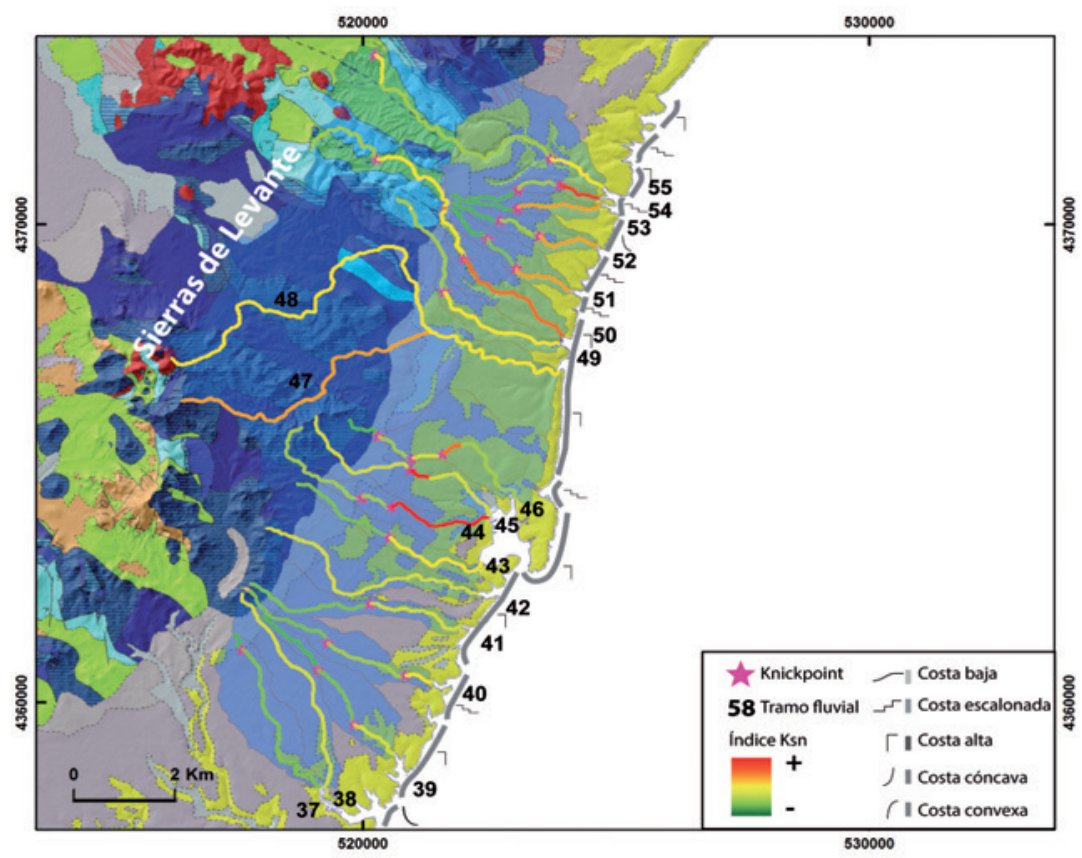

Figura 9. Mapa geológico de la zona 6 (fuente: IGME) correspondiente a los ríos que inciden en la parte central y superior del Levante de Mallorca, con los trazados de la red fluvial y su gradación según su índice de $K_{s n}$, la localización de los knickpoints y el tipo de costa. El lector puede remitirse a la leyenda del mapa del IGME. 
Asociado al contacto Cuaternario-Tortoniense se desarrolla un knickpoint en 13 de los 18 tramos. En 7 de los 12 tramos con menor área de drenaje situada en las Sierras se encuentra un knickpoint, cerca de la cabecera, sin que exista ninguna estructura o cambio litológico. Este hecho podría ser causado al alcanzar el área de drenaje mínima para que el río provoque incisión y la aparición de una nueva tipología de knickpoint por este fenómeno podría asociarse con, como mínimo, otros 10 tramos de las zonas anteriormente analizadas.

Por ello es necesario analizar específicamente los perfiles longitudinales para constatar la existencia de dicha área mínima para este contexto litológico y climatológico, que se mantiene prácticamente invariable en toda la zona de estudio.

\subsection{Perfiles longitudinales de los tramos fluviales}

La representación gráfica de los perfiles longitudinales de elevación-distancia se ha realizado también separando las diferentes zonas con el fin de observar o confirmar patrones de forma (Fig. 10). Se observa que dentro de cada zona los perfiles tienen características comunes tales como distancia de la cabecera al nivel de base, pendiente media y rango de valores de concavidad. Aunque se ha establecido una separación en zonas es inevitable observar como los perfiles evolucionan de un extremo al otro, provocando por una parte similitudes entre tramos contiguos separados en dos zonas distintas. Por otra parte esto aporta información sobre el carácter transicional de cada zona.

Un análisis en detalle permite observar las siguientes características y tendencias. En la zona 1 , los perfiles inicialmente se acortan y aumentan su pendiente para luego alargarse y disminuir su pendiente hacia la zona 2, en un recorrido horario de longitud-elevación. La zona 2 sigue aumentando la pendiente, disminuye la longitud de los perfiles y se pierde la concavidad entrando en el campo de perfiles muy convexos. En la zona 3 disminuye la convexidad terminando en perfiles ya cóncavos, la pendiente disminuye y se aprecia un ligero aumento en la longitud de los tramos. En la zona 4 se llega a la longitud máxima de los tramos y la mínima pendiente; predomina la concavidad. En la zona 5 aparecen tramos mixtos cóncavo-convexos que indican que los ríos transcurren por tramos con diferente contexto e historia geológica, diferenciados con parámetros de estabilidad y de inestabilidad (concavidad y convexidad). La pendiente media tiende a aumentar y la longitud inicialmente disminuye para finalmente volver a aumentar. En la zona 6 predominan tramos de muy poca concavidad en los tramos cortos, la pendiente media tiende a aumentar.

En el análisis entre zonas se observa que en términos de pendiente media, factor que influye directamente en el potencial erosivo de los ríos, se sitúa en una zona de valores medios los tramos de la zona 1, aumentando considerablemente hasta un máximo en la zona 2.

Posteriormente los ríos pierden pendiente en la zona 3 llegando a un mínimo en la zona 4. Finalmente aumenta en las zonas 5 y 6 . 

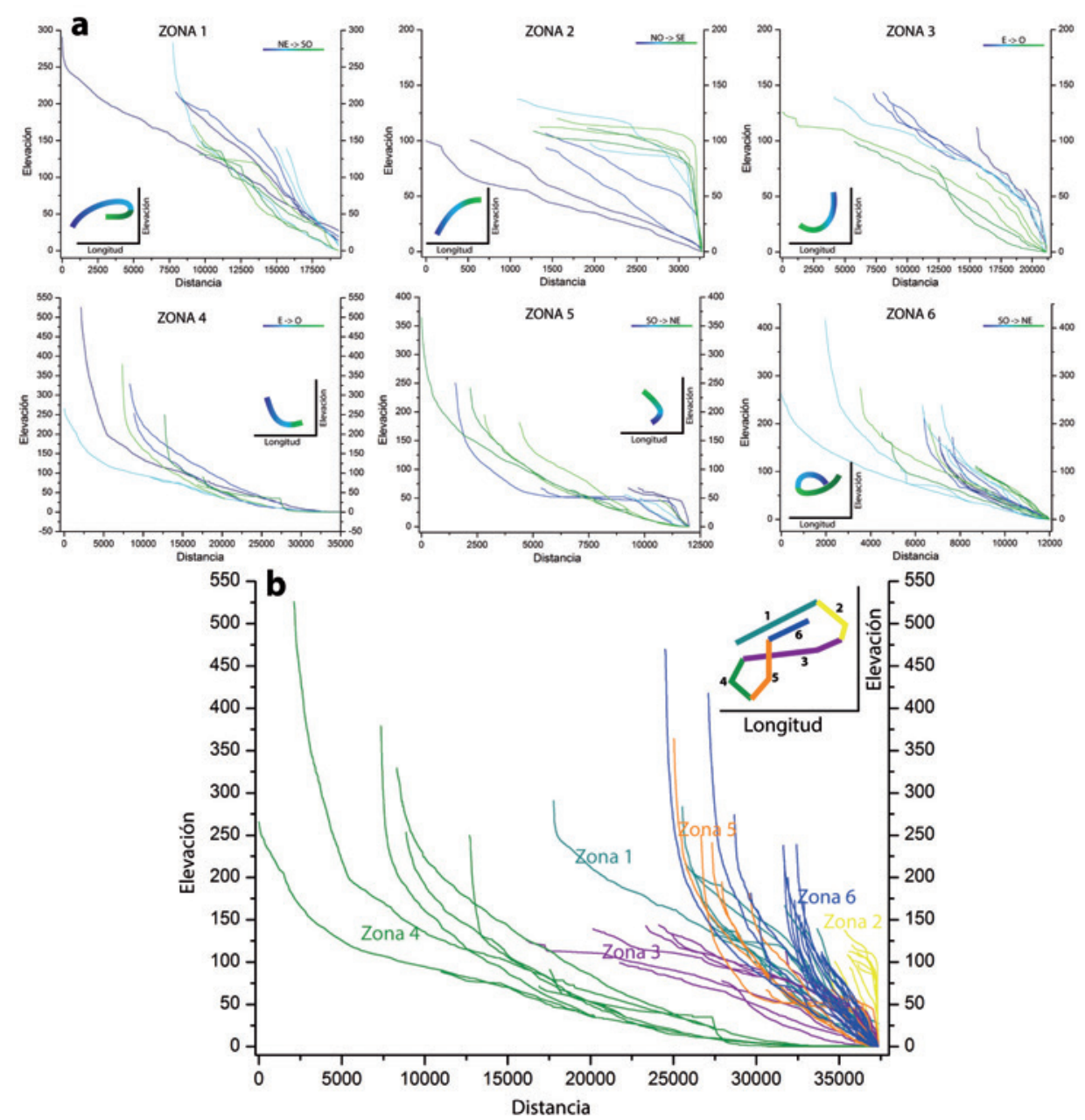

Figura 10.a) Representación gráfica por zonas de los perfiles longitudinales de todos los tramos fluviales analizados. De azul a verde su distribución geográfica dentro de cada zona y en detalle su evolución espacial respecto a su longitud y elevación. b) Representación de todos los perfiles en un mismo origen y por zonas según color. En detalle la evolución espacial entre zonas respecto a su longitud y elevación.

\subsection{Knickpoints asociados a un área de drenaje mínima}

Tras haber detectado los knickpoints en zonas cercanas a una cabecera situada en una zona llana, se han recogido los valores de área de drenaje en cada punto donde se detecta el knickpoint (Tabla 1). Esto permite identificar un área de drenaje mínima a partir de la cual se desarrollan knickpoints. Se observa en la distribución de los valores que existe un pequeño rango de área de drenaje donde se sitúan la mayoría de ellos indicando que puede existir un área mínima para que se produzca erosión por escorrentía con- 
centrada para un mismo contexto fisiográfico, con unas condiciones climatológicas y litológicas. Esta área mínima tiene un valor medio de $4.7 \times 10^{5} \mathrm{~m}^{2}$ para la zona de estudio, siendo el $95 \%$ de los knickpoints con áreas de drenaje entre $2.3 \times 10^{5} \mathrm{~m}^{2}$ y $7.2 \times 10^{5} \mathrm{~m}^{2}$. En un estudio de 246 knickpoints activos en cascadas de Nueva Zelanda, Crosby y Whipple (2006) determinaron que aproximadamente el 70\% de los knickpoints se localizaban en áreas de drenaje entre $1 \times 10^{5} \mathrm{~m}^{2}$ y $1 \times 10^{6} \mathrm{~m}^{2}$. En la isla de Jura, Escocia, Castillo et al. (2013) fijan un umbral de $<2 \mathrm{~km}^{2}$ de área de drenaje para que sea posible el retroceso de knickpoints.

Tabla 1. Listado de los valores de área de drenaje aguas arriba en los puntos donde se ha localizado un knickpoint cerca de una cabecera llana. Valor de media, desviación estándar y los límites inferior y superior donde se concentra el $95 \%$ de los valores.

\begin{tabular}{|c|c|c|c|}
\hline Tramo & KP área $\left(\mathbf{K m}^{2}\right)$ & Tramo & KP área $\left(\mathbf{k m}^{2}\right)$ \\
\hline 2 & 0.36 & 26 & 0.10 \\
\hline 6 & 0.52 & 27 & 0.55 \\
\hline 7 & 0.06 & 30 & 0.10 \\
\hline 9 & 0.16 & 31 & 0.17 \\
\hline 11.4 & 0.50 & 32 & 0.07 \\
\hline 11.3 & 0.57 & 40 & 0.37 \\
\hline 11.1 & 0.69 & 41 & 0.40 \\
\hline 19 & 0.39 & 46 & 0.09 \\
\hline 21 & 0.18 & 51 & 0.23 \\
\hline 56 & 0.48 & 52 & 0.12 \\
\hline 23 & 2.21 & 53 & 0.13 \\
\hline 24 & 0.16 & 54 & 0.14 \\
\hline 62 & 2.68 & 55 & 0.07 \\
\hline 63 & 1.45 & & Límite superior 95\% km²) \\
\hline Media $\left(\mathrm{km}^{2}\right)$ & Desviación $\left(\mathrm{km}^{2}\right)$ & Límite inferior $95 \%\left(\mathrm{~km}^{2}\right)$ & 0.72 \\
\hline 0.47 & 0.63 & 0.23 & \\
\hline
\end{tabular}

\section{Conclusiones}

La red de drenaje del sur y sureste de la isla de Mallorca está condicionada en su forma, distribución y características por diversos factores. Sin estar asociada a ningún orógeno ni atravesando grandes zonas de topografía montañosa, la pendiente regional y la topografía de algunas superficies distribuyen los cauces y definen su pendiente hacia las zonas más deprimidas o costeras. Los cursos fluviales, en general, tienen menor pendiente cuanta mayor es su área de drenaje. Su estado de equilibrio dinámico es mayor cuanta mayor es su área de drenaje siendo pues más cóncavos, con menor índice de pen- 
diente y con menor presencia de knickpoints y de menor salto. En tramos de menor longitud y área de drenaje, su respuesta ante las perturbaciones con el fin de alcanzar un equilibrio es más lenta, viéndose reflejados de forma más clara patrones de convexidad elevados, índices de pendiente muy superiores a la media de la zona y una mayor presencia de knickpoints y de mayor entidad. Esto hace que la zona estudiada, con una clara homogeneidad litológica, climática y en un contexto tectónico regional aparentemente inactivo, presente extremos en el grado de equilibrio y madurez de los cursos fluviales. Esto plantea la necesidad de descubrir y cuantificar qué papel juegan los factores que, a priori, parecían despreciables como las variaciones eustáticas Plio-Cuaternarias, la neotectónica y los procesos kársticos.

El análisis e interpretación de los 74 perfiles longitudinales de los ríos localizados en el sur y sureste de Mallorca revela la presencia de knickpoints de origen litológico en 31 de los 37 tramos donde el río atraviesa el contacto entre los materiales Pliocenos o Cuaternarios y las calcarenitas del Tortoniense-Mesiniense. Esto es debido al contraste de respuesta ante agentes erosivos, principalmente fenómenos de disolución y karst.

En los tramos donde su cabecera no está situada en áreas de cierto relieve, sino que aparecen de una escorrentía situada en una superficie relativamente llana, existe un área de drenaje mínima para que se produzca erosión por escorrentía concentrada para el clima semiárido correspondiente al sur y sureste de Mallorca y una misma litología. El $95 \%$ de los knickpoints de este origen se encuentran entre los valores de $2.3 \times 10^{5} \mathrm{y}$ $7.2 \times 10^{5} \mathrm{~m}^{2}$.

Se ha comprobado que la utilización de perfiles heredados o paleoperfiles es útil para determinar bajadas del nivel eustático o saltos de falla bajo determinadas condiciones. En un tramo al sur de la zona de estudio se ha registrado un salto de $30 \mathrm{~m}$ que puede indicar un movimiento vertical de bloques.

\section{Referencias}

Allen, P.A. 2008. From landscapes into geological history. Nature 451, 274-276.

Ahnert, F. 1970. Functional relationships between denudation, relief, and uplift in large mid-latitude drainage basins. American Journal of Science 268, 243-263.

Bishop, P. 2007. Long-term landscape evolution: linking tectonics and surface processes. Earth Surface Processes and Landforms 32, 329-365.

Bishop, P., Goldrick, G. 2000. Geomorphological evolution of the East Australian continental margin. En Geomorphology and Global Tectonics, M.A. Summerfield (ed.), John Wiley, Chichester, pp. 227-255.

Castillo, M., Bishop, P., Jansen, J.D. 2013. Knickpoint retreat and transient bedrock channel morphology triggered by base-level fall in small bedrock river catchments: The case of the isle of Jura, Scotland. Geomorphology 180-181, 1-9.

Crosby, B.T., Whipple, K.X. 2007. Knickpoint initiation and distribution within fluvial networks: 236 waterfalls in the Waipaoa River, North Island, New Zealand. Geomorphology 82, 16-38.

Dietrich, W.E., Bellugi, D.G., Sklar, L.S., Stock, J.D., Heimsath, A.M., Roering, J.J. 2003. Geomorphic transport laws for predicting landscape form and dynamics. En Prediction in Geomorphology, P.R. Wilcock, R.M. Iverson (eds.), Geophysical Monograph 135, AGU, pp. 103-132. 
Duvall, A., Kirby, E., Burbank, D. 2004. Tectonic and lithologic controls on bedrock channel profiles and processes in coastal California. Journal of Geophysical Research 109, F02002. doi:10.1029/2003JF000086.

England, P.C., Molnar, P. 1990. Surface uplift, uplift of rocks, and exhumation of rocks. Geology $18,1173-1177$.

Finlayson, D.P., Montgomery, D.R., Hallet, B. 2002. Spatial coincidence of rapid inferred erosion with young metamorphic massifs in the Himalayas. Geology 30, 219-222.

Finnegan, N.J., Roe, G., Montgomery, D.R., Hallet, B. 2005. Controls on the cannel width of rivers: implications for modeling fluvial incision of bedrock. Geology 33, 229-232.

Gardner, T.W. 1983. Experimental study of knickpoint and longitudinal evolution in cohesive, homogeneous material. Geological Society of America Bulletin 94, 664-672.

Gelabert, B. 1998. La estructura geológica de la mitad occidental de la Isla de Mallorca. Colección Memorias, Instituto Tecnológico GeoMinero de España, Madrid, 129 pp.

Gelabert, B., Balaguer, P., Fornós, J.J., Gómez-Pujol, L. 2007. El papel de la estructura en la formación y evolución de un acantilado costero del sureste de Mallorca (Illes Balears). En Geomorfología Litoral: Migjorn y Llevant de Mallorca, J.J. Fornós, J. Ginés, L. Gómez-Pujol (eds.), Monografies de la Societat d'Història Natural de les Balears 15, pp. 125-134.

Goldrick, G., Bishop, P. 2007. Regional analysis of bedrock stream long profiles: evaluation of Hack's SL form, and formulation and assessment of an alternative (the DS form). Earth Surface Processes and Landforms 32, 649-671.

Grimalt-Gelabert, M., Rodríguez-Perea, A. 1989. Cabals màxims al Llevant i Migjorn de Mallorca durant les revingudes del setembre de 1989. Treballs de Geografia 42, 7-18.

Hack, J.T. 1957. Studies of longitudinal stream profiles in Virginia and Maryland. United States Geological Survey Professional Paper 294, 45-94.

Hovius, N. 2000. Macro-scale process systems of mountain belt erosion. En Geomorphology and Global Tectonics, M.A. Summerfield (ed.), John Wiley and Sons, Chichester, pp. 77-105.

Howard, A.D., Dietrich, W.E., Seidl, M.A. 1994. Modeling fluvial erosion on regional to continental scales. Journal of Geophysical Research-Solid Earth 99, 13971-13986.

Hutchinson, M. F. 1989. A new procedure for gridding elevation and stream line data with automatic removal of spurious pits. Journal of Hydrology 106, 211-232.

Montgomery, D.R., Brandon, M.T. 2002. Topographic controls on erosion rates in tectonicallyactive mountain ranges. Earth and Planetary Science Letters 201, 481-489.

Roering, J.J., Kirchner, J.W., Dietrich, W.E. 1999. Evidence for nonlinear, diffusive sediment transport on hillslopes and implications for landscape morphology. Water Resources Rese$\operatorname{arch} 35,853-870$.

Rosselló i Verger, V.M. 2007. Cales, torrents, fractures i carst a Mondragó (com a pretext). En Geomorfologia litoral i Quaternari, G.X. Pons, D. Vicens (eds.), Monografies de la Societat d'Història Natural de les Balears 14, pp. 287-298.

Snow, R.S., Slingerland, R.L. 1987. Mathematical modelling of graded river profiles. Journal of Geology 95, 15-33.

Summerfield, M.A. 1991. Global Geomorphology. Longman, London.

Whipple, K.X. 2001. Fluvial landscape response time: how plausible is steady-state denudation? American Journal of Science 301, 313-325.

Whipple, K.X. 2004. Bedrock rivers and the geomorphology of active orogens. Annual Review of Earth and Planetary Sciences 32, 151-185.

Whipple, K.X., Tucker, G.E. 1999. Dynamics of the stream-power river incision model: implications for height limits of mountain ranges, landscape response timescales, and research needs. Journal of Geophysical Research 104, 17661-17674. 
Whipple, K.X., DiBiase, R., Crosby, B. 2011. Bedrock rivers. En Treatise on Geomorphology, J. Shroder, H. Wohl (ed.), Academic Press, SanDiego, CA, pp. 550-573.

Wobus, C.W., Hodges, K.V., Whipple, K.X. 2003. Has focused denudation sustained active thrusting at the Himalayan topographic front? Geology 10, 861-864.

Wobus, C., Whipple, K.X., Kirby, E., Snyder, N., Johnson, J., Spyropolou, K., Crosby, B., Sheehan, D. 2006. Tectonics from topography: procedures, promise, and pitfalls. En Tectonics, Climate, and Landscape Evolution, S.D. Willett, N. Hovius, M.T. Brandon, D. M. Fisher (eds.), Geological Society of America Special Paper, vol. 398, pp. 55-74. 